\title{
Dynamical Friction in Disk Galaxies: The Radial Dependence
}

\author{
Mattias Wahde \\ Division of Mechanics, Chalmers University of Technology, 41296 \\ Göteborg, Sweden \\ Karl Johan Donner \\ Observatory, University of Helsinki, Finland
}

\begin{abstract}
A simple formula is derived for the force of dynamical friction acting on a satellite galaxy which is moving on a nearly circular orbit around its parent galaxy. Using this formula, estimates of the decay time are computed. The results are then compared with the corresponding results from numerical simulations, and are found to be in good agreement.
\end{abstract}

\section{Introduction and Motivation}

When a satellite galaxy orbits around its parent galaxy, it will lose angular momentum through dynamical friction, and eventually spiral in towards the center of the parent galaxy. For the case of a satellite moving in the plane of the disk, it can be shown (Wahde, Donner, \& Sundelius 1995) that the frictional force from the disk dominates over that from the halo if the disk is more massive than approximately 10 per cent of the total mass of the parent galaxy.

The angular momentum exchange between a disk and a satellite can be estimated using the formula derived by Lynden-Bell \& Kalnajs (1972), in which the friction is given as a sum over resonant contributions. Using first order epicyclic orbits for the disk stars, only the corotation resonance and the $l= \pm 1, m$ Lindblad resonances contribute (see below). The contribution from the corotation resonance dominates the friction, with the Lindblad resonances contributing approximately 10 per cent (Donner \& Sundelius 1993). The effects of the velocity dispersion can be estimated using higher order epicyclic orbits, and it can be shown (Wahde, Donner, \& Sundelius 1995) that these effects are not very important. Thus, it can be expected that the corotation contribution alone will give a sufficiently accurate value of the frictional force.

In this paper we will consider the orbital decay of a satellite moving on an initially circular prograde orbit in the plane of the disk. If the satellite is not too massive, the orbit of the infalling satellite can be considered to be a sequence of circular orbits at progressively smaller radii. 


\section{Theoretical Calculation of the Orbital Decay}

The angular momentum exchange between a disk and a satellite can be computed as a sum of resonant terms (Lynden-Bell \& Kalnajs 1972):

$$
\dot{L}=2 \pi^{3} \sum_{l, m=-\infty}^{\infty} \int_{0}^{\infty} \int_{0}^{\infty} m\left(l \frac{\partial F}{\partial J}+m \frac{\partial F}{\partial L}\right)\left|\Psi_{l m}\right|^{2} \delta\left(l \Omega_{r}+m \Omega_{\varphi}-\omega\right) d J d L .
$$

where $F$ denotes the disk distribution function, $\Psi_{l m}$ the coefficients of the Fourier expansion of the potential, and $\omega / m$ the orbital frequency of the satellite. The frequencies $\Omega_{r}$ and $\Omega_{\varphi}$ reduce in the epicyclic approximation to the epicyclic frequency $(\kappa)$ and the angular frequency $(\Omega)$, respectively. For a satellite which is modelled as a softened point mass, the corotation contribution $(l=0)$ at radius $r$ can be written

$$
\dot{L}_{c r}=\frac{G^{2} M_{s}^{2}}{4 r A} \frac{d}{d r}\left(\frac{\Sigma}{B}\right)\left(\frac{r}{\epsilon}\right)^{2}
$$

where $G$ is the gravitational constant, $M_{s}$ the satellite mass, $A, B$ the (absolute values of the) Oort constants, $\Sigma$ the surface density of the disk, and $\epsilon$ the softening parameter of the satellite. If the infall process is sufficiently slow, we can consider the satellite to be, at any given moment, on a circular orbit and write the angular momentum as

$$
L=M_{s} r v_{c}(r)
$$

where $v_{c}(r)$ is the circular velocity at radius $r$. Using this equation we can compute $\dot{L}$ and, with the help of equation (2), obtain an implicit expression for $r$ as a function of time:

$$
\int_{r_{0}}^{r} \frac{4 r A\left(v_{c}(r)+r v_{c}^{\prime}(r)\right) d r}{G^{2} M_{s} \frac{d}{d r}\left(\frac{\Sigma}{B}\right)\left(\frac{r}{\epsilon}\right)^{2}}=\int_{t_{0}}^{t} d t=t-t_{0}
$$

The derivation given above neglects the self-gravity of the disk. For the disksatellite configuration considered in this paper, the self-gravity of the disk has been shown to give only a modest enhancement of the frictional force (Donner \& Sundelius 1993; Wahde, Donner, \& Sundelius 1995), and it will be neglected here.

Throughout this paper we will use a galaxy model with a Plummer bulge $\left(r_{b}=0.5, m_{b}=0.1\right)$, a Plummer halo $\left(r_{h}=10.0, m_{h}=1.5\right)$ and an exponential disk $\left(r_{d}=3.0, m_{d}=0.5\right)$. The units are such that $G$, the gravitational constant, equals unity, the unit of mass is $2 \times 10^{11} M_{\odot}$, the unit of length is $1 \mathrm{kpc}$, and the unit of time is $1.05 \mathrm{Myr}$.

As can be seen from equation (4) the decay time varies as the square of the softening parameter $\epsilon$. The choice of the softening parameter for a real satellite galaxy is a difficult problem. A reasonable upper bound is provided by the Roche radius of the satellite

$$
r_{\text {roche }}=\left(\frac{G M_{s}}{4 A^{2}}\right)^{\frac{1}{3}}
$$

Here we will take the softening parameter to be equal to the Roche radius. 


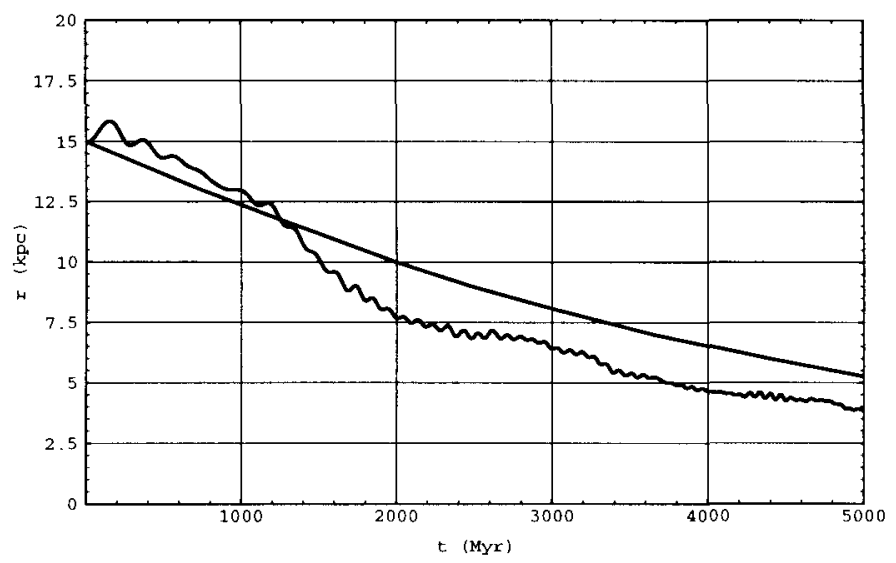

Figure 1. Orbital decay for a satellite of mass 0.006 .

\section{Simulations and Comparison with Theory}

In order to test the theory discussed above we have carried out numerical simulations of the orbital decay process. We have used a restricted three-body code in which the disk particles move under the influence of the halo-bulge-disk potential (which is constant in time) and the satellite potential. 'The satellite is influenced by the halo-bulge potential as well as the individual particles making up the disk. The satellite mass lies in the range $[0.001,0.01]$ i.e. from 0.2 up to 2.0 per cent of the disk mass. For smaller satellites, properties not considered in this paper such as, for instance, the thickness of the disk, must be taken into account. For larger satellites the orbit can no longer be considered as a sequence of circular orbits. Furthermore, the effects on the disk will be large and a bar may form, under which circumstances the theory is not valid. In Figure 1 the orbital decay of a satellite of mass 0.006 is plotted. The smooth curve is the theoretical estimate, obtained through equation (4), whereas the ragged curve shows the results from a numerical simulation. An important issue in most numerical simulations is the number of particles. In the orbital decay simulations described in this paper, it is the disk particles that give rise to the frictional force on the satellite. If the disk is composed of only very few particles (a few thousand, say), the satellite will experience a noisy force, and the simulations cannot be trusted. Our experiments show that at least 30,000 particles are necessary in order to obtain a smooth enough force.

\section{References}

Donner, K. J. \& Sundelius, B. 1993, MNRAS, 265, 88

Lynden-Bell, D. \& Kalnajs, A. J. 1972, MNRAS, 157, 1

Wahde, M., Donner, K. J., \& Sundelius, B. 1995, MNRAS, submitted 